\title{
The Application of Off-Axis Electron Holography to Electrically Biased Single GaN Nanowires for Electrical Resistivity Measurement
}

\author{
S. Yazdi ${ }^{1}$, T. Kasama ${ }^{1}$, M. Beleggia ${ }^{1}$, R. Ciechonski ${ }^{2}$, O. Kryliouk ${ }^{3}$ and J.B. Wagner ${ }^{1}$ \\ ${ }^{1}$ Center for Electron Nanoscopy, Technical University of Denmark, DK 2800 Kgs. Lyngby, Denmark \\ ${ }^{2}$ GLO-AB, Ideon Science Park, Lund, Sweden \\ ${ }^{3}$ GLO-USA, Sunnyvale, CA, USA
}

Semiconductor nanowires hold promise to become building blocks for the next generation of electronic and optoelectronic devices, mainly due to their nanoscale geometry. However, the geometry of nanowires has made it highly challenging to measure their optical and electrical characteristics [1]. Here, we report a method based on off-axis electron holography for measuring the electrical resistivity of single nanowires.

Electron holography enables us to measure the shift induced in the phase of the electron beam as passed through a transmission electron microscope (TEM) specimen. This phase shift, $\varphi(x, y)$, is proportional to the distribution of electrostatic potentials, $V(x, y, z)$, within the specimen. In the absence of dynamical diffraction and magnetic field, and if it is assumed that the electrostatic potential variation along the beam direction, $z$, is negligible, the relation between the phase shift and the electrostatic potential can be simplified to:

$$
\varphi(x, y)=C_{E} V(x, y) \cdot t(x, y)
$$

where $C_{E}$ is a constant depending on the accelerating voltage of the microscope and $t(x, y)$ represents the specimen thickness.

A nanowire with the length of $L$, cross-sectional area of $A$ and electrical resistivity of $\rho$ would have the electrical resistance of $R=\rho L / A$. If the current $I$ is passed through this nanowire, the potential drop along $\Delta L$ length of the nanowire would be:

Substituting equation (2) into (1) results in:

$$
\Delta V=\rho \frac{\Delta L}{A} I
$$

$$
\Delta \varphi=C_{E} \rho \frac{\Delta L}{A} I . t
$$

Therefore, the phase shift measured along a nanowire using electron holography increases linearly with the current, and the resistivity of nanowire can be extracted from the slope of this linear relationship.

A single GaN nanowire, with $2 \mu \mathrm{m}$ length, $9.8 \times 10^{-10} \mathrm{~cm}^{2}$ hexagonal cross-sectional area and $10^{19} \mathrm{~cm}^{-3}$ nominal dopant concentration, was detached and lifted up from its substrate in-situ in a scanning electron microscope (SEM) (Fig. 1a), and placed on two pre-patterned W plates (Fig. 1b). Off-axis electron holograms were acquired as a function of biasing current using an FEI Titan 80-300 TEM operated at $300 \mathrm{kV}$ and an Aduro biasing specimen holder [2]. The phase image obtained without biasing was used as a reference for the measurement of the phase change along the nanowire. The contribution of electric field leakage to the vacuum, from the electrical contacts between the nanowire and $\mathrm{W}$ plates to the phase shift, was estimated from the phase profiles in the vacuum (arrow B in Fig. b) and subtracted from the phase profile along the nanowire at each current. The resistivity of the nanowire was measured to be $(8 \pm 1) \times 10^{-4} \Omega$-cm. [3]

References

[1] S Kristian et al., Nature Nanotechnology 7 (2012), p. 718. 
[2] Protochips, Inc.; http://www.protochips.com

[3] The authors acknowledge funding from 'Nanowires for solid state lighting'. NMP.2011.2.2-3, Project ID: 280773

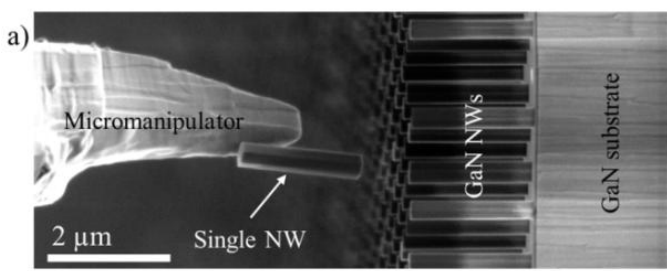

Figure 1: Specimen preparation. a) Detaching a single nanowire from its substrate in an SEM using a micromanipulator and placing it down on a prepatterned SiN membrane (b).

b)

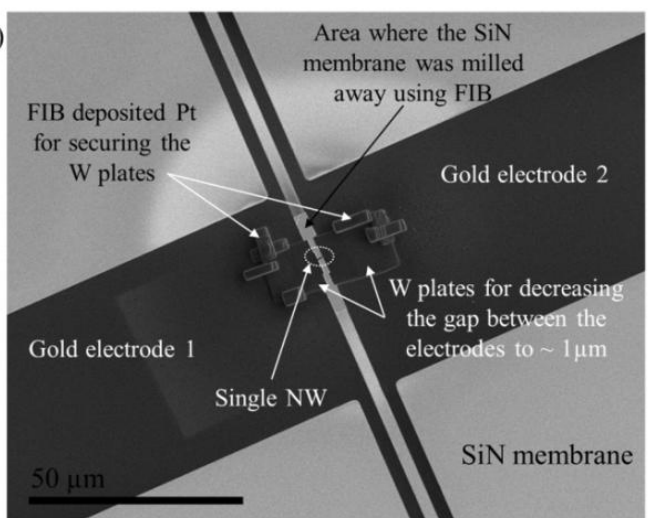

b) SEM image showing a $2 \mu \mathrm{m}$ nanowire sitting on a $1 \mu \mathrm{m}$ gap in between two $\mathrm{W}$ plates. The gap was micromachined on a $\mathrm{W}$ lamella using focus ion beam milling. The SiN membrane was partially milled away to provide the required vacuum region for electron holography.

a)

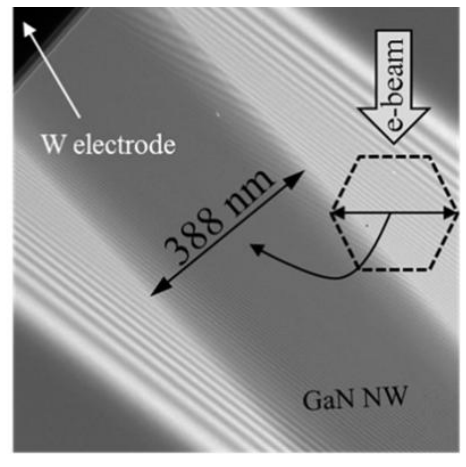

b)

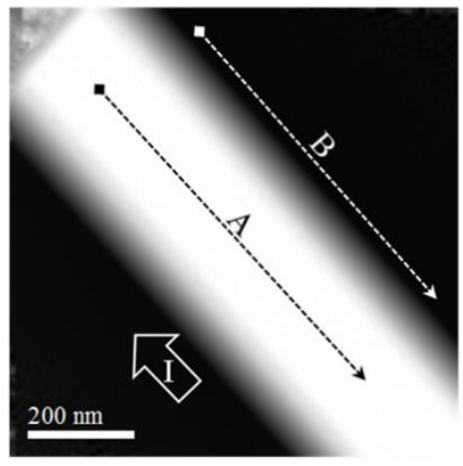

c)

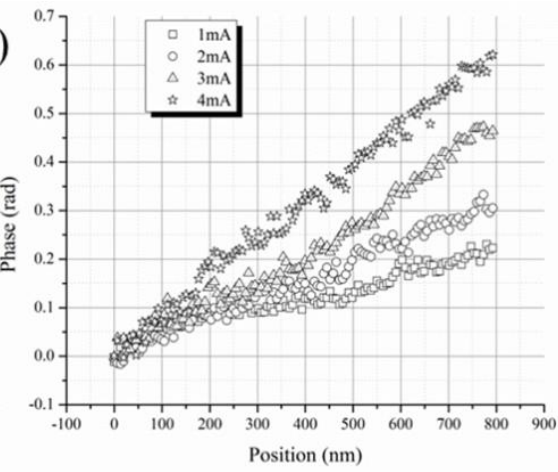

d)

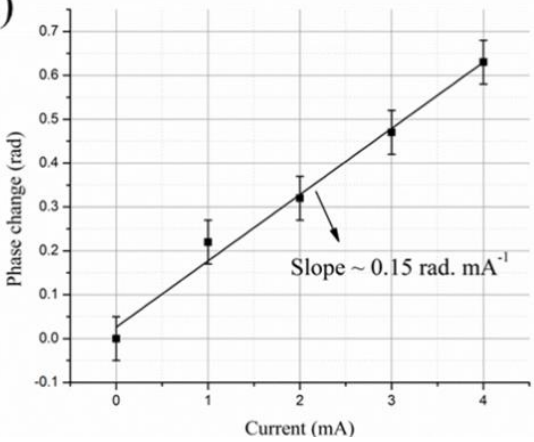

Figure 2: a) Hologram acquired from a GaN nanowire. The nanowire thickness was measured directly from the hexagonal geometry of nanowire. b) Reconstructed phase image. At each biasing current, the phase profile in a vacuum (arrow B) was subtracted from the phase profile along the nanowire (arrow A), to compensate for the leakage of electric fields from the electrical contacts to the vacuum. Arrow I shows the direction of the applied current. c) Corrected phase profiles as a function of biasing current. d) Phase differences measured along $800 \mathrm{~nm}$ of the nanowire as a function of current 